\title{
Can Complete Axillary Node Dissection Be Safely Omitted in Patients with Early Breast Cancer When the Sentinel Node Biopsy Is Positive for Malignancy? An Update for Clinical Practice
}

\author{
RITIKA GERA, ABDUL KASEM and KEFAH MOKBEL
}

The London Breast Institute, Princess Grace Hospital, London, U.K.

\begin{abstract}
The sentinel lymph node biopsy (SLNB) has become the new standard-of-care for patients with clinically node-negative invasive breast cancer. The focused examination of fewer lymph nodes in addition to improvements in histopathological and molecular analysis have increased the rate at which micrometastases and isolated tumor cells are identified. We reviewed the literature and summarized the evidence regarding the need for complete axillary lymph node dissection (ALND) following the finding of a positive sentinel node biopsy through the identification of the most important outcomes and evaluation of quality of evidence. The article focuses on the safe omission of complete ALND when the axillary lymph nodes contain macrometastases and provides an overview of the topic primarily based on level 1 evidence derived from randomized clinical trials with a critical appraisal of the ACOSOG Z0011 trial.
\end{abstract}

Sentinel lymph node biopsy (SLNB) was pioneered for the staging of melanoma in 1994 (1). Shortly thereafter, Giuliano et al. (2) demonstrated the feasibility of SLNB for breast cancer. Over the subsequent two decades, the SLNB has been validated by several trials, and has become the new standard-of-care for patients with clinically node-negative

This article is freely accessible online.

Correspondence to: Kefah Mokbel, The London Breast Institute, The Princess Grace Hospital, 42-52 Nottingham Place, London W1U5NY, U.K. Tel: +44 2079082040, Fax: +44 2079082275, e-mail: kefahmokbel@hotmail.com

Key Words: Sentinel lymph node biopsy, early breast cancer, axillary radiotherapy, axillary dissection, evidence-based medicine, clinical practice update, review. invasive breast cancer. Since the SLNB is found to be positive in approximately $30 \%$ of patients undergoing surgical treatment of clinically node-negative breast cancer, $70 \%$ of women are now able to avoid radical surgery in the form of complete axillary node dissection (ALND), which is known to be associated with a higher incidence of morbidity, longer hospitalisation, and impairment of quality of life (3).

The optimal SLNB technique utilises a radioactive isotope tracer in addition to a blue dye and when both modalities are used and the procedure is performed by an experienced surgeon, it has been reported to be highly accurate (4). It was noted in early studies that there was not any identifiable advantage of lymphoscintigraphy mapping even for surgeons learning the techniques. Intra-operative frozen-section analysis of the sentinel node has been shown to be accurate for the evaluation of metastatic disease with high sensitivity and excellent specificity $(5,6)$.

Since the eighth edition of the American Joint Committee on Cancer/Union for International Cancer Control TNM classification (7) the extent of metastatic disease in the sentinel node has been classified into three categories: isolated tumour cells (ITCs; $<2 \mu \mathrm{m}$, not larger than $0.2 \mathrm{~mm}$ in largest dimension); micrometastatic disease (MM; $2 \mu \mathrm{m}-2 \mathrm{~mm}$ ); and macrometastatic disease $(>2 \mathrm{~mm})$. In addition, the eighth edition also mentions real-time polymerase chain reaction as a new modality for detecting cancerous cells in sentinel nodes.

The aim of this review was to analyze and summarise the existing literature and evidence to assess the need for complete ALND post positive SLNB identification. Level 1 evidence from critically appraised randomised clinical trials was used.

\section{Management of ITCs and MM}

ITCs and MM have become more common findings due to the increased use of immunohistochemistry (IHC). Similarly, new refinements in real-time polymerase chain reaction, such 
as One-Stop Nodal Analysis, have been recently introduced for intra-operative evaluation, and have been demonstrated to be even more sensitive for detecting metastatic deposits than IHC (8).

The clinical implications of these findings were unclear, with little progress towards a consensus until very recently, due to the non-availability of suitable level evidence (9-11). Although the presence of ITCs in the sentinel nodes has a prognostic significance, there is a consensus that it does not represent an indication for further treatment of the axilla (11). The presence of micrometastatic disease in the sentinel node was, until recently, considered an absolute indication for a complete ALND (12). However, more recent evidence is highly suggestive that a more conservative approach to such microdeposits would be more appropriate despite modest upstaging of the disease (9). A recent randomised controlled trial (RCT) confirmed the oncological safety of omitting complete ALND for MM-positive SLNB (IBCSG 23-01) (13).

\section{Management of Macro-metastases}

The presence of macro-metastases in the sentinel nodes is considered a routine indication for complete ALND, as stated by the National Institute for Health and Care Excellence (2018) (14). However, a recent RCT carried out by the American College of Surgeons Oncology Group (Z0011) showed that in women undergoing breast-conserving surgery (BCS) for clinically node-negative $\mathrm{T} 1 / \mathrm{T} 2$ invasive breast cancer, complete ALND is not required if only 1-2 sentinel nodes were found to be invaded by malignancy. The five-year disease-free survival and overall survival were similar in the ALND group versus that without ALND. There was no significant difference between the two groups in relation to the primary tumour characteristics and the use of adjuvant systemic therapy. All patients received adjuvant radiotherapy following BCS (15). Therefore, patients who fulfil the criteria for this trial can avoid complete ALND, which is associated with a higher incidence of complications. Patients must: be at least 18 years old, have sentinel node-treated clinical T1/T2 N0 M0 breast cancer, have negative lumpectomy margins, have undergone sentinel node dissection within 60 days of their invasive breast carcinoma diagnosis, and have an Eastern Cooperative Oncology Group/Zubrod status of 2 or less. The presence or absence of extra-capsular extension (ECE) was not analysed in this trial. A recent retrospective study showed that the presence of an ECE greater than $2 \mathrm{~mm}$ in the sentinel nodes was associated with significant tumour burden in non-sentinel nodes during ALND and therefore this feature can be added to the eligibility criteria for omitting ALND when the SLNB is positive (i.e. ECE <2 mm) (16).

Schwentner et al. used data from 342 patients with breast cancer identified as having clinically node-negative disease with a positive SLNB and subsequent ALND (16). In their retrospective cohort study, the following characteristics were identified regarding extracapsular nodal extension-positive patients: a significantly higher proportion with three or more positive ALNs, significantly greater lymph node metastasis size of more than $2 \mathrm{~mm}$, and lower rates of T1 status. It should be noted that there was variation in the results depending on the type of analysis performed on the data. With univariate analysis, extracapsular nodal extension was related to worse overall survival. With multivariate analysis, nodal status, age and comorbidities were adjusted for so this effect disappeared. The authors suggested that extracapsular nodal extension will only influence patients with a higher number of positive nodes, in which case it acts as one of the most predictive parameters for breast cancer survival outcomes.

Complete ALND is still indicated in women who are undergoing BCS, found to have metastatic disease involving three or more lymph nodes, or undergoing total mastectomy with positive SLNB involving any lymph nodes.

In a further shift towards less radical surgery of the axilla, the recent AMAROS RCT showed that axillary radiotherapy was as effective as complete ALND in terms of 5-year overall and disease-free survival. Furthermore, radiotherapy (50 Gy in 25 fractions) was associated with a lower incidence of lymphoedema of the arm, in the short and long term. The authors, however, observed a non-significant trend towards impairment in shoulder movement in women undergoing radiotherapy to the axilla in the short term. Earlier analysis of the trial data showed that the lack of knowledge of the pathological status of the non-sentinel nodes did not influence the treatment decisions in relation to adjuvant systemic therapy (17). Moreover, oncologists are increasingly basing their systemic treatment recommendations (18) on the use of multigene molecular signatures of the primary tumour, such as Mammaprint ${ }^{\circledR}$, Oncotype DX ${ }^{\circledR}$, and Endopredict ${ }^{\circledR}$.

The American Society of Clinical Oncology (ASCO) has recently updated its guidelines to reflect the results of these trials (19). The adoption of these guidelines worldwide will spare thousands of women radical surgery to the axilla without compromising their clinical outcome. However, the final treatment recommendations must always be made in the context of a multidisciplinary setting. The findings of these trials are consistent with the biological behaviour of breast cancer and the longstanding perception that axillary surgery aims to provide staging and prognostic information to guide systemic treatment and radiotherapy recommendations rather than achieving mechanical eradication of the disease. Evidence-based medicine means that we should embrace the results of these RCTs and introduce the new ASCO recommendations into our clinical practice to improve the quality of life of our patients. The main limitation of the initial report of the Z0011 trial was the short follow-up duration of 5 years and this has limited the worldwide adoption of the trial results in clinical practice since hormonesensitive breast cancer tends to relapse after 5 years of treatment. 


\section{The 10-Year Update of the ACOSOG Z0011 Trial}

The Z0011 investigators updated their results in 2017 and reported the clinical outcome 10 years after the original trial was conducted (20). The findings showed that at a median follow-up of 9.3 years, SLNB was non-inferior to ALND in both overall and disease-free survival. Hence, they concluded that routine use of ALND is not indicated in patients who fulfil the trial's criteria for SLNB.

The lack of significant difference in disease-free survival between patients treated with SLNB as opposed to ALND suggests that nodal recurrence is primarily an early event. Although hormone-receptor positive tumours are known to carry a higher risk of recurrence during extended follow-up $(21,22)$, the patients who underwent SLND in the Z0011trial did not have a significantly greater rate of recurrence when compared to those who underwent ALND. It should be noted that in an adjusted analysis, the elimination of ALND was not associated with better overall survival but the use of SLNB was.

The treatment of patients who fit the criteria set out by the Z0011 trial has changed considerably. Overall, the use of ALND has decreased dramatically and the use of SLNB has also increased. In the Z0011 study, after controlling for various other confounding factors, age was not significantly associated with an increase in the rate of recurrence and ALND also does not need to be recommended in younger women or women with triple-negative breast cancer. Nevertheless, caution should be exercised when applying the results of the Z0011 trial to those patients under-represented in the trial, such as young patients, and patients with triplenegative breast cancer, grade 3 tumours and infiltrating lobular carcinoma, and the decision should be always made in the context of a multidisciplinary discussion.

The use and omission of nodal irradiation along with the use of high-tangent field irradiation was similarly distributed by treatment group in this trial, hence it was unlikely to have affected the outcome of the study. Recent studies have investigated the effect of nodal irradiation on long-term survival and reached differing results $(23,24)$. It should be noted that the aforementioned studies examined different types of breast cancer (high-risk early- stage versus stage II-III).

The following issues are acknowledged by the researchers of the Z0011 trial: The low accrual and event rate mean that the study did not reach its pre-specified target sample size of 1,900 participants nor 500 deaths, not all biological subtypes were represented in large numbers, different individual circumstances may have resulted in differences in outcome, and some patients (evenly distributed amongst the two groups) had variations in irradiation protocol which carried the potential of slightly altering outcomes.

The results of their follow-up indicate that the use of ALND can no longer be justified in women who meet the criteria set out by the Z0011 trial, particularly if they are postmenopausal and have hormone-sensitive breast cancer. However, it is unsafe to eliminate ALND for women who do not meet these criteria.

\section{A Critical Appraisal of the ACOSOG Z0011 Trial}

The following is taken from an analysis of the limitations present in multiple RCTs examining axillary treatment in primary breast cancer and low burden axillary disease (25).

A hazard ratio of 1.3 was calculated by the authors of the ACOSOG Z0011 trial and was used to justify the conclusion that SLNB is non-inferior to ALND. It is a very large margin for determining whether SLNB is non-inferior and this makes it surprising that the study did not meet its recruitment goals. The low recruitment rate raises concerns of high recruitment bias. It also means that the researchers did not achieve $90 \%$ statistical power as was their aim, instead achieving approximately $35 \%$. Of the original patients, $19.4 \%$ were lost to follow-up, and although there was no significant loss of patients in one trial group compared to the other, it can only have further hampered the conclusion drawn from their data. Furthermore, 103 ineligible patients were included in the trial; this was $11.6 \%$ of the total patient cohort; $3.7 \%$ of patients undergoing SLNB had three or more positive lymph nodes, which the researchers originally claimed to be part of their exclusion criteria.

Contrary to what is claimed by the Z0011 trial researchers, disease characteristics at baseline were not well balanced between the two arms of the trial; the ALND group carried a greater burden of nodal disease. A two-tailed chi-squared test with one degree of freedom examining the difference in patients with MM between the two groups gives a value of $p=0.046$, which indicates borderline significance. The inclusion of patients with MM and ITCs undermined the purpose of the trial because these patients were comparatively unlikely to die. In addition, this could have been a contributing factor to the early closure of the trial.

The trial committed major protocol violations. Too many patients were assigned to the wrong type of surgery to reasonably suggest that this was due to chance; $7.6 \%$ of patients randomised to ALND underwent SLNB, whereas $2.5 \%$ had ALND when they were assigned to SLNB. These patients were then included in all analyses. Furthermore, radiotherapy details were missing in $29.3 \%$ patients. Despite radiotherapy being administered reasonably equally across both treatment arms, the effect of radiotherapy post-ALND versus the effect on an untreated axilla (essentially an axilla treated with SLNB) is very different and may have significantly affected the endpoints. Patients receiving nodal radiotherapy had significantly more involved lymph nodes, which indicates that its use was not random and was targeted according to physician selection. A total of $16.5 \%$ of the 
patients who underwent SLNB were also treated with supraclavicular fossa radiotherapy. These violations suggest that SLNB patients received effective localised treatment which negates any comparative conclusion drawn between SLNB and ALND.

Finally, most patients were postmenopausal and had hormone-sensitive breast cancer. This weakens the evidence for women younger than 45 years of age and those with other subtypes such as triple-negative and human epidermal growth factor receptor 2 (HER2)-positive breast cancer. However, most patients with triple-negative or HER2-positive breast cancer are nowadays treated with neoadjuvant systemic therapy and this point is discussed later in this article.

Therefore, caution should be exercised when concluding that SLNB is non-inferior to ALND based on this trial alone due to flaws present in the study design and execution. Moreover, the results of the Z0011trial are not consistent with those of the MA20 trial data (26), which showed that combined regional radiotherapy and ALND improved disease outcome compared to standalone ALND.

Another study examined the relationship between patient age and tumour subtype and the extent of axillary surgery conducted on the patients in the Z0011 trial (27). The authors concluded that in all groups, the use of SLNB alone increased over time, but the extent to which a patient had axillary surgery differed according to their age and tumour subtype. Logistical regression was used to analyze the data. Patients who were 70 years of age or older were significantly likelier to undergo SLNB alone. Younger patients (older than 40 years but younger than 70 years) and patients with HER2-positive/triple-negative disease were significantly less likely to undergo SLNB alone compared to patients with luminal subtypes.

\section{Can SLNB Be a Viable Alternative to Complete ALND and Following Neoadjuvant Chemotherapy (NAC) in Women with Node-Positive Cancer?}

We recently performed a meta-analysis (28) using data from 19 articles and 3,398 biopsy-proven node-positive patients to conclude that SLNB, following NAC, has a false-negative rate (FNR) of $13 \%$, a sentinel lymph node identification rate (SLNIR) of $91 \%$, and an adjusted pathological complete response (pCR) of $47 \%$. The SLNB is, therefore, a viable alternative to complete ALND provided that at least two nodes are sampled and there is a radiological evidence of response to NAC. However, more refined patient selection and better choice of optimal techniques can improve the SLNIR and FNR.

Optimum mapping techniques might reduce FNR but in this analysis, there was no consensus between studies on mapping techniques. Some techniques have shown a promising reduction of FNR, such as marking the pathological ALN with radioactive iodine seeds (MARI) or marker clips to permit its removal in a process similar to SLNB. This allows FNR to fall to between 0 to $7 \%$, which is at least similar if not better than that for the SLNB for clinically node-negative patients (28). The SLNIR of $91 \%$ found in our meta-analysis is still lower than those found in meta-analyses conducted on node-negative patients. The relatively high estimate of $47 \%$ pCR shown in this meta-analysis suggests that ALND may be overtreatment for these node-positive patients if performed routinely and SLNB after neoadjuvant therapy can lead to omission of ALND in a significant proportion of patients who are nodepositive prior to the initiation of therapy, resulting in reduced morbidity (especially lymphoedema) and hospitalization and better quality of life for patients. Sampling of more than one sentinel node seems to improve the SLNBIR and reduce the FNR (29) and the authors of the meta-analysis recommended sampling at least two sentinel nodes post neoadjuvant therapy.

Axillary ultrasound scanning can clinically stage axillary nodes at the time of diagnosis of breast cancer and can also be used post-NAC where it is better at detecting positive nodes instead of predicting pCR (30). In determining ALN status, positron-emission tomography (PET) has a high specificity range but a low and broad sensitivity range (31, 32). Larger studies are required to appropriately assess the sensitivity and specificity of magnetic resonance scanning for imaging axillary nodes post-NAC.

This meta-analysis admitted several limitations. Nodepositive patients were selected "with or without needle biopsy", hence six out of the 19 studies included did not use a biopsy of ALNs pre-NAC to determine histopathology. The clinical complete nodal response covered a large range, from $21.5 \%$ to $83.9 \%$. The pooled estimate for FNR was found to be $13 \%$, which is lower than the results found by other meta-analyses and higher than what is found in nodenegative patients. However, the FNR was reasonably low here so it could be considered as an option for node-positive patients. Publication bias may be present due to the lack of inclusion of non-English studies. The use of a randomeffects model may lessen the reliability of results. Not all studies reported receptor status. Moreover, a high degree of heterogeneity was observed between studies.

\section{Can the Use of SLNB and ALND Be Omitted Entirely in Patients with Node-positive Disease Post Neoadjuvant Systemic Therapy (NST)? A Summary}

It should be noted that the following arguments are based on the findings of one article alone and that they do not compare the use of SLNB versus the MARI procedure (33). In this study, the MARI node is defined as an iodine-125 seed placed under ultrasound guidance in the largest tumourpositive axillary node. 
Ninety-three patients were used to examine whether tailored axillary treatment post-NST using the MARI procedure and ${ }^{18}$ F-fluorodeoxyglucose (FDG) PET-computed omography (CT) would be sufficient to avoid using the standard of care (ALND). After the MARI procedure, all patients underwent ALND to allow estimation of potential over- and undertreatment. It was found that $74 \%$ patients could have avoided ALND, three patients were potentially undertreated, and $16 \%$ patients were potentially overtreated. The MARI identification rate was $97 \%$ with $7 \%$ FNR. It was also minimally invasive compared to ALND.

Axillary treatment was omitted in patients with one-three FDG-avid ALNs present on a PET/CT scan and a tumournegative MARI node. Patients who did not meet these criteria were offered axillary treatment. Proof that $95 \%$ of patients were treated correctly would be acceptable evidence for a change of practice. Patients with invasive breast cancer larger than $3 \mathrm{~cm}$ in diameter and/or at least one tumourpositive ALN were offered NST, which was administered under institutional guidelines.

Currently, there are two trials which are investigating axillary treatment post-NST in clinically node-positive patients which should provide information on the long-term follow up when ALND or radiotherapy is omitted.

pCR is defined as the 'absence of vital tumour cells in the MARI node and additional nodes in the ALND, irrespective of the response in the breast'; this may exclude vital evidence which is reflective of the treatment effectiveness. Patients with a pCR of the primary tumour/axilla must be identified for the surgical procedure to have any comparative benefit in locoregional control.

The total patient cohort used in this summary of 93 is relatively too small for the researchers to be able to make treatment recommendations. The MARI node had been in situ for a median of 18 weeks at the time of removal. In the Netherlands, the country where the study was conducted, the cost of FDG PET-CT is much lower compared to other countries around the world. Patients with one to three suspected ALNs and a false-negative MARI, or patients in whom the PET-CT scanning underestimated axillary staging, would be undertreated.

\section{Conclusions and Recommendations for Clinical Practice}

Complete ALND is not routinely indicated if the SLNB is positive for ITCs or MM. In the presence of macrometastases, the SLNB alone is non-inferior to complete ALND in patients with T1-T2 undergoing BCS and adjuvant radiation according to the mature RCT with 10 years of follow-up (ACOSOG Z0011) (20). Considering the limitations of the ACOSOG Z0011 trial and the need for further confirmatory studies, the decision to omit complete ALND should be made after multidisciplinary discussion of the final pathology. This approach seems to be safe in postmenopausal women with hormone-sensitive breast cancer who fulfil the Z0011 eligibility criteria supported by level 1 evidence. Caution should be exercised when applying this to young women (younger than 45 years) and those with basal phenotypes. There is a retrospective evidence (34) that ALND could also be safely omitted in patients with a macrometastasis-positive SLNB who were under-represented in the ACOSOG Z0011 trial including T3 tumours, more than two positive sentinel nodes, invasive lobular carcinoma, oestrogen receptor-negative status, extranodal extension, Nottingham grade 3 , or age $<50$ years. However, larger cohorts and longer follow-up times are needed to conclusively determine if ALND exclusion is safe for these patients.

In patients with biopsy-proven ALN involvement undergoing neoadjuvant chemotherapy, SLNB is a valid alternative to complete ALND provided that at least two nodes are sampled and there is a radiological evidence of an excellent response to NAC. However, more refined patient selection and better choice of optimal techniques can improve the SLNIR and FNR.

When complete ALND is indicated, axillary radiotherapy can be considered as a valid alternative with equivalent outcome.

\section{References}

1 Morton DL, Wen DR, Wong JH, Economou JS, Cagle LA, Storm FK, Foshag LJ and Cochran AJ: Technical details of intraoperative lymphatic mapping for early stage melanoma. Arch Surg 127(4): 392-399, 1992.

2 Giuliano AE, Kirgan DM, Guenther JM and Morton DL: Lymphatic mapping and sentinel lymphadenectomy for breast cancer. Ann Surg 220(3): 391-398; discussion 391-398, 1994.

3 Veronesi U, Paganelli G, Viale G, Luini A, Zurrida S, Galimberti V, Intra M, Veronesi P, Maisonneuve P, Gatti G, Mazzarol G, De Cicco C, Manfredi G and Fernandez JR: Sentinel-lymph-node biopsy as a staging procedure in breast cancer: Update of a randomised controlled study. Lancet Oncol 7(12): 983-990, 2006.

4 Posther KE, McCall LM, Blumencranz PW, Burak WE, Beitsch PD, Hansen NM, Morrow M, Wilke LG, Herndon JE, Hunt KK, and Giuliano AE: Sentinel node skills verification and surgeon performance: Data from a multicenter clinical trial for earlystage breast cancer. Ann Surg 242(4): 593-599; discussion 593$599,2005$.

5 Morrow M, Rademaker AW, Bethke KP, Talamonti MS, Dawes LG, Clauson J and Hansen N: Learning sentinel node biopsy: Results of a prospective randomized trial of two techniques. Surgery 126(4): 714-720; discussion 720-722, 1999.

6 Ali R, Hanly AM, Naughton P, Castineira CF, Landers R, Cahill RA and Watson RG: Intraoperative frozen section assessment of sentinel lymph nodes in the operative management of women with symptomatic breast cancer. World J Surg Oncol 6: 69, 2008.

7 Hortobagyi GN, Connolly JL, D’Orsi CJ, Edge SB, Mittendorf EA, Rugo HS, Solin LJ, Weaver DL, Winchester DJ and Giuliano A: AJCC Cancer Staging Manual. Eighth Edition. American College of Surgeons, Chicago, IL, USA, 2016. 
8 Chaudhry A, Williams S, Cook J, Jenkins M, Sohail M, Calder C, Winters ZE and Rayter Z: The real-time intra-operative evaluation of sentinel lymph nodes in breast cancer patients using one-step nucleic acid amplification (OSNA) and implications for clinical decision-making. Eur J Surg Oncol 40(2): 150-157, 2014.

9 Patani $\mathrm{N}$ and Mokbel K: The clinical significance of sentinel lymph node micrometastasis in breast cancer. Breast Cancer Res Treat 114(3): 393-402, 2009.

10 Salhab M, Patani N and Mokbel K: Sentinel lymph node micrometastasis in human breast cancer: An update. Surg Oncol 20(4): e195-e206, 2011.

11 Patani N and Mokbel K: Clinical significance of sentinel lymph node isolated tumour cells in breast cancer. Breast Cancer Res Treat 127(2): 325-334, 2011.

12 ASCO Guideline Recommendations for Sentinel Lymph Node Biopsy in Early-Stage Breast Cancer: Guideline Summary. J Oncol Pract 1(4): 134-136, 2005.

13 Galimberti V, Cole BF, Zurrida S, Viale G, Luini A, Veronesi P, Baratella $\mathrm{P}$, Chifu C, Sargenti M, Intra M, Gentilini O, Mastropasqua MG, Mazzarol G, Massarut S, Garbay JR, Zgajnar J, Galatius H, Recalcati A, Littlejohn D, Bamert M, Colleoni M, Price KN, Regan MM, Goldhirsch A, Coates AS, Gelber RD, Veronesi U and International Breast Cancer Study Group Trial 23-01 investigators: Axillary dissection versus no axillary dissection in patients with sentinel-node micrometastases (IBCSG 23-01): a phase 3 randomised controlled trial. Lancet Oncol 14(4): 297-305, 2013.

14 Nice.org.uk: Early and locally advanced breast cancer: diagnosis and management I Guidance and guidelines I NICE. [online] Available at: https://www.nice.org.uk/guidance/ng101/chapter/ Recommendations\#surgery-to-the-breast, 2008.

15 Giuliano AE, McCall L, Beitsch P, Whitworth PW, Blumencranz P, Leitch AM, Saha S, Hunt KK, Morrow M and Ballman K: Locoregional recurrence after sentinel lymph node dissection with or without axillary dissection in patients with sentinel lymph node metastases: The American College of Surgeons Oncology Group Z0011 Randomized Trial. Ann Surg 252(3): 426-432, 2010.

16 Schwentner L, Dayan D, Wockel A, Janni W, Kreienberg R, Blettner $\mathrm{M}$ and Wollschlager D: Is extracapsular nodal extension in sentinel nodes a predictor for nonsentinel metastasis and is there an impact on survival parameters? A retrospective single center cohort study with 324 patients. Breast J 24(4): 480-486, 2018.

17 Donker M, van Tienhoven GV, Straver ME, Meijnen P, van de Velde CJ, Mansel RE, Cataliotti L, Westenberg AH, Klinkenbijl JH, Orzalesi L, Bouma WH, van der Mijle HC, Nieuwenhuijzen GA, Veltkamp SC, Slaets L, Duez NJ, de Graaf PW, van Dalen T, Marinelli A, Rijna H, Snoj M, Bundred NJ, Merkus JW, Belkacemi Y, Petignat P, Schinagl DA, Coens C, Messina CG, Bogaerts $\mathrm{J}$ and Rutgers EJ: Radiotherapy or surgery of the axilla after a positive sentinel node in breast cancer (EORTC 1098122023 AMAROS): a randomised, multicentre, open-label, phase 3 non-inferiority trial. Lancet Oncol 15(12): 1303-1310, 2014.

18 El Hage Chehade H, Wazir U, Mokbel K, Kasem A and Mokbel $\mathrm{K}$ : Do online prognostication tools represent a valid alternative to genomic profiling in the context of adjuvant treatment of early breast cancer? A systematic review of the literature. Am J Surg 215(1): 171-178, 2018.
19 Lyman GH, Temin S, Edge SB, Newman LA, Turner RR, Weaver DL, Benson AB, Bosserman LD, Burstein HJ, Cody H, Hayman J, Perkins CL, Podoloff DA, Giuliano AE and American Society of Clinical Oncology Clinical Practice: Sentinel lymph node biopsy for patients with early-stage breast cancer: American Society of Clinical Oncology clinical practice guideline update. J Clin Oncol 32(13): 1365-1383, 2014.

20 Giuliano AE, Ballman KV, McCall L, Beitsch PD, Brennan MB, Kelemen PR, Ollila DW, Hansen NM, Whitworth PW, Blumencranz PW, Leitch AM, Saha S, Hunt KK and Morrow M: Effect of axillary dissection vs. no axillary dissection on 10-year overall survival among women with invasive breast cancer and sentinel node metastasis: The ACOSOG Z0011 (Alliance) Randomized Clinical Trial. JAMA 318(10): 918-926, 2017.

21 Colleoni M, Sun Z, Price KN, Karlsson P, Forbes JF, Thurlimann B, Gianni L, Castiglione M, Gelber RD, Coates AS and Goldhirsch A: Annual hazard rates of recurrence for breast cancer during 24 years of follow-up: Results from the International Breast Cancer Study Group Trials I to V. J Clin Oncol 34(9): 927-935, 2016.

22 Pan H, Gray RG, Davies C, Peto R, Bergh JCS, Pritchard KI, Dowsett M, Hayes DF and Mustacchi G: Predictors of recurrence during years 5-14 in 46,138 women with ER+ breast cancer allocated 5 years only of endocrine therapy (ET). J Clin Oncol 34 (Suppl 15): 505, 2016.

23 Moreno AC, Lin YH, Bedrosian I, Shen Y, Stauder MC, Smith BD, Buchholz TA, Babiera GV, Woodward WA and Shaitelman SF: Use of regional nodal irradiation and its association with survival for women with high-risk, early stage breast cancer: A National Cancer Database analysis. Adv Radiat OncoI 2(3): 291300, 2017.

24 Chang JS, Park W, Kim YB, Lee IJ, Keum KC, Lee CG, Choi $\mathrm{DH}$, Suh $\mathrm{CO}$ and Huh SJ: Long-term survival outcomes following internal mammary node irradiation in stage II-III breast cancer: results of a large retrospective study with 12-year follow-up. Int J Radiat Oncol Biol Phys 86(5): 867-72, 2013.

25 Robertson JF, Herrod PJ, Matthew J, Kilburn L, Coles CE and Bradbury I: Treatment of the axilla in patients with primary breast cancer and low burden axillary disease: Limitations of the evidence from randomised controlled trials. Crit Rev Oncol Hematol 110: 74-80, 2017.

26 Whelan TJ, Olivotto IA, Parulekar WR, Ackerman I, Chua BH, Nabid A, Vallis KA, White JR, Rousseau P, Fortin A, Pierce LJ, Manchul L, Chafe S, Nolan MC, Craighead P, Bowen J, McCready DR, Pritchard KI, Gelmon K, Murray Y, Chapman JA, Chen BE, Levine MN and MA.20 Study Investigators: Regional nodal irradiation in early-stage breast cancer. N Engl J Med 373(4): 307-316, 2015.

27 Ong CT, Thomas SM, Blitzblau RC, Fayanju OM, Park TS, Plichta JK, Rosenberger LH, Hyslop T, Shelley EH and Greenup RA: Patient age and tumor subtype predict the extent of axillary surgery among breast cancer patients eligible for the American College of Surgeons Oncology Group Trial Z0011. Ann Surg Oncol 24(12): 3559-3566, 2017.

28 El Hage Chehade H, Headon H, El Tokhy O, Heeney J, Kasem A and Mokbel K: Is sentinel lymph node biopsy a viable alternative to complete axillary dissection following neoadjuvant chemotherapy in women with node-positive breast cancer at diagnosis? An updated meta-analysis involving 3,398 patients. Am J Surg 212(5): 969-981, 2016. 
29 Kuehn T, Bauerfeind I, Fehm T, Fleige B, Hausschild M, Helms G, Lebeau A, Liedtke C, von Minckwitz G, Nekljudova V, Schmatloch S, Schrenk P, Staebler A and Untch M: Sentinellymph-node biopsy in patients with breast cancer before and after neoadjuvant chemotherapy (SENTINA): a prospective, multicentre cohort study. Lancet Oncol 14(7): 609-618, 2013.

30 Alvarado R, Yi M, Le-Petross H, Gilcrease M, Mittendorf EA, Bedrosian I, Hwang RF, Caudle AS, Babiera GV, Akins JS, Kuerer HM and Hunt KK: The role for sentinel lymph node dissection after neoadjuvant chemotherapy in patients who present with node-positive breast cancer. Ann Surg Oncol 19(10): 3177-3184, 2012.

31 Liang X, Yu J, Wen B, Xie J, Cai Q and Yang Q: MRI and FDGPET/CT based assessment of axillary lymph node metastasis in early breast cancer: a meta-analysis. Clin Radiol 72(4): 295-301, 2017.

32 Kumar R, Zhuang H, Schnall M, Conant E, Damia S, Weinstein S, Chandra P, Czerniecki B and Alavi A: FDG PET-positive lymph nodes are highly predictive of metastasis in breast cancer. Nucl Med Commun 27(3): 231-236, 2006
33 Koolen BB, Donker M, Straver ME, van der Noordaa MEM, Rutgers EJT, Valdes Olmos RA and Vrancken Peeters MJTFD: Combined PET-CT and axillary lymph node marking with radioactive iodine seeds (MARI procedure) for tailored axillary treatment in node-positive breast cancer after neoadjuvant therapy. Br J Surg 104(9): 1188-1196, 2017.

34 Gebhardt BJ, Thomas J, Horne ZD, Champ CE, Farrugia DJ, Diego E, Ahrendt GM and Beriwal S: Is completion axillary lymph node dissection necessary in patients who are underrepresented in the ACOSOG Z0011 trial? Adv Radiat Oncol 3(3): 258-264, 2018.

Received July 23, 2018

Revised September 16, 2018

Accepted September 19, 2018 